\title{
AN EXPLICIT FORMULA FOR THE LINEARIZATION COEFFICIENTS OF BESSEL POLYNOMIALS
}

\author{
MOHAMED JALEL ATIA AND JIANG ZENG
}

\begin{abstract}
We prove a single sum formula for the linearization coefficients of the Bessel polynomials. In two special cases we show that our formula reduces indeed to Berg and Vignat's formulas in their proof of the positivity results about these coefficients (Constructive Approximation, 27 (2008), 15-32). As a bonus we also obtain a generalization of an integral formula of Boros and Moll (J. Comput. Appl. Math. 106 (1999), 361-368).
\end{abstract}

Keywords Bessel polynomials, Linearization coefficients, Integral formula of Boros and Moll.

A.M.S. Classification: Primary 33C10; Secondary 60E05

\section{INTRODUCTION}

The Bessel polynomials $q_{n}$ of degree $n$ are defined by

$$
q_{n}(u)=\sum_{k=0}^{n} \frac{(-n)_{k} 2^{k}}{(-2 n)_{k} k !} u^{k}
$$

where we use the Pochhammer symbol $(z)_{n}:=z(z+1) \ldots(z+n-1)$ for $z \in \mathbb{C}$ and $n \in \mathbb{N}$. The first values are

$$
q_{0}(u)=1, \quad q_{1}(u)=1+u, \quad q_{2}(u)=1+u+\frac{u^{2}}{3} .
$$

Using hypergeometric functions, we have $q_{n}(u)={ }_{1} F_{1}(-n ;-2 n ; 2 u)$. They are normalized according to $q_{n}(0)=1$, and thus differ from the monic normalization $\theta_{n}(u)$ in Grosswald's monograph [6]:

$$
\theta_{n}(u)=\frac{(2 n) !}{n ! 2^{n}} q_{n}(u)
$$

The polynomials $\theta_{n}$ are sometimes called the reverse Bessel polynomials and $y_{n}(u)=u^{n} \theta_{n}\left(\frac{1}{u}\right)$ the ordinary Bessel polynomials. These Bessel polynomials are, then, written as

$$
y_{n}(u)=\frac{(2 n) !}{n ! 2^{n}} u^{n} q_{n}\left(\frac{1}{u}\right)=\sum_{k=0}^{n} \frac{(n+k) !}{2^{k} k !(n-k) !} u^{k} .
$$


The so-called linearization coefficients $\beta_{k}^{(n, m)}$ of the Bessel polynomials [2] are defined by

$$
q_{n}(a u) q_{m}((1-a) u)=\sum_{k=0}^{n+m} \beta_{k}^{(n, m)}(a) q_{k}(u) .
$$

For example, we have $\beta_{k}^{(3,5)}=0$ for $k=0,1,2$ and

$$
\begin{aligned}
& \beta_{3}^{(3,5)}(a)=a^{7}, \\
& \beta_{4}^{(3,5)}(a)=7 a^{7}(1-a), \\
& \beta_{5}^{(3,5)}(a)=(1-a)^{2}\left(84 a^{6}-126 a^{5}+126 a^{4}-84 a^{3}+36 a^{2}-9 a+1\right), \\
& \beta_{6}^{(3,5)}(a)=\frac{11}{5}(1-a)^{3}\left(126 a^{4}-168 a^{3}+108 a^{2}-36 a+5\right), \\
& \beta_{7}^{(3,5)}(a)=\frac{143}{5} a^{2}(1-a)^{4}\left(12 a^{2}-9 a+2\right), \\
& \beta_{8}^{(3,5)}(a)=143 a^{3}(1-a)^{5} .
\end{aligned}
$$

Recently Berg and Vignat [2, 3] have established some important results about the coefficients $\beta_{k}^{(n, m)}(a)$ with applications. In particular, they proved [2, Theorem 2.1] that $\beta_{n}^{(n, 0)}(a)=a^{n}$ and, for $0 \leq k \leq n-1$,

$$
\beta_{k}^{(n, 0)}(a)=a^{k}(1-a) \frac{\left(\begin{array}{l}
n \\
k
\end{array}\right)}{\left(\begin{array}{l}
2 n \\
2 k
\end{array}\right)} \sum_{j=0}^{(n-j-1) \wedge k}\left(\begin{array}{c}
n+1 \\
k-j
\end{array}\right)\left(\begin{array}{c}
n-k-1 \\
j
\end{array}\right)(1-a)^{j} .
$$

Moreover, for $n, m \geq 1$, Berg and Vignat [2] have proved that the coefficients $\beta_{k}^{(n, m)}(a)$ satisfy the following recurrence relation [2, Lemma 3.6]:

$$
\frac{1}{2 k+1} \beta_{k+1}^{(n, m)}(a)=\frac{a^{2}}{2 n-1} \beta_{k}^{(n-1, m)}(a)+\frac{(1-a)^{2}}{2 m-1} \beta_{k}^{(n, m-1)}(a),
$$

for $k=0,1, \ldots, m+n-1$ and $\beta_{0}^{(n, m)}(a)=0$. From (4) and (5) they derive the positivity of $\beta_{k}^{(n, m)}(a)$ when $0 \leq a \leq 1$ and also that $\beta_{k}^{(n, m)}(a)=$ 0 for $k<\min (m, n)$. However, an explicit single sum formula for $\beta_{k}^{(n, m)}(a)$ is missing in their paper.

Our main result is an explicit single sum formula for $\beta_{i}^{(n, m)}(a)$, which provides actually the unique solution of the recurrence system (5) with the boundary condition (4).

Theorem 1. For $k=0,1, \ldots, n+m$, we have

$$
\begin{aligned}
\beta_{k}^{(n, m)}(a) & =a^{2 n+m-k}(1-a)^{-n+k} \frac{(1 / 2)_{n+m-k}(1 / 2)_{k}}{(1 / 2)_{n}(1 / 2)_{m}} \\
& \times \sum_{j=0}^{2(n+m-k)}(-1)^{j}\left(\begin{array}{c}
n+m+1 \\
2 n+2 m-2 k-j
\end{array}\right)\left(\begin{array}{c}
-m+k+j \\
j
\end{array}\right) a^{-j} .
\end{aligned}
$$


Equivalently, the coefficients $\beta_{k}^{(n, m)}(a)$ can be written in terms of hypergeometric function:

(i) if $k \geq\lceil(n+m-1) / 2\rceil$, then

$$
\begin{aligned}
\beta_{k}^{(n, m)}(a) & =a^{2 n+m-k}(1-a)^{-n+k} \frac{(1 / 2)_{n+m-k}(1 / 2)_{k}}{(1 / 2)_{n}(1 / 2)_{m}}\left(\begin{array}{c}
n+m+1 \\
2 n+2 m-2 k
\end{array}\right) \\
& \times{ }_{2} F_{1}\left(\begin{array}{c}
-2 m-2 n+2 k,-m+k+1 \\
2 k-m-n+2
\end{array} ; \frac{1}{a}\right) ;
\end{aligned}
$$

(ii) if $k \leq\lfloor(n+m-1) / 2\rfloor$, then

$$
\begin{aligned}
\beta_{k}^{(n, m)}(a) & =a^{n+k+1}(1-a)^{-n+k} \frac{(1 / 2)_{n+m-k}(1 / 2)_{k}}{(1 / 2)_{n}(1 / 2)_{m}}\left(\begin{array}{c}
n-k-1 \\
n+m-2 k-1
\end{array}\right) \\
& \times(-1)^{n+m-1}{ }_{2} F_{1}\left(\begin{array}{c}
-m-n-1, \quad n-k \\
n+m-2 k
\end{array} ; \frac{1}{a}\right) .
\end{aligned}
$$

We first derive some immediate consequences from the above theorem. Assume that $n \leq m$ and $0 \leq a \leq 1$.

(1) If $k<n$, then the binomial coefficient $\left(\begin{array}{c}n-k-1 \\ n+m-2 k-1\end{array}\right)$ vanishes because $n+m-2 k-1>n-k-1$. Thus formula (8) implies that $\beta_{k}^{(n, m)}(a)=0$ for $k<m \wedge n$.

(2) If $k \geq n$, then each term in (66) is a polynomial in $a$. Indeed, it is clear that $(1-a)^{-n+k} \in \mathbb{Z}[a]$, and

- if $k \geq m$, then $2 n+m-k-j \geq 2 n+m-k-2(n+m-k)=$ $k-m \geq 0$ for $0 \leq j \leq 2(n+m-k)$,

- if $k<m$, then the binomial coefficient $\left(\begin{array}{c}-m+k+j \\ j\end{array}\right)$ does not vanish only if $-m+k+j<0$, that is, $j<m-k$, therefore, $2 n+m-k-j>2 n+m-k-(m-i)=2 n \geq 0$.

(3) From definition (3) we derive immediately the symmetry property:

$$
\beta_{k}^{(n, m)}(a)=\beta_{k}^{(m, n)}(1-a) .
$$

This identity follows also from (7) (resp. (8)) and Pfaff's transformation (see [1, p. 94]):

$$
{ }_{2} F_{1}\left(\begin{array}{cc}
A B & B \\
C & ; x
\end{array}\right)=(1-x)^{-A}{ }_{2} F_{1}\left(\begin{array}{cc}
A, & C-B \\
& C
\end{array} \frac{x}{x-1}\right),
$$

with $A=-2 n-2 m+2 k, B=-m+k+1, C=-n-m+2 k+2$ and $x=1 / a$ if $k \geq\lceil(n+m-1) / 2\rceil$ (resp. $A=-n-m-1$, $B=n-k, C=n+m-2 k$ and $x=1 / a$ if $k \leq\lfloor(n+m-1) / 2\rfloor)$.

We shall prove Theorem 1 in Section 2. As applications of our Theorem 1, we shall derive a formula of Berg and Vignat [2] when $m=n$, a positivity result as well as a formula generalizing an integral evaluation of Boros and Moll [4] in Section 3.

\section{Proof of Theorem 1}

We shall first derive a double sum formula for the coefficients $\beta_{i}^{(n, m)}(a)$. 
2.1. A double sum. Assume that $m \geq n$. Let

$$
\alpha_{k}^{(n)}=\frac{(-n)_{k} 2^{k}}{(-2 n)_{k} k !} \text {. }
$$

By definition (11) we have

$$
q_{n}(a u) q_{m}((1-a) u)=\sum_{k=0}^{m+n} u^{k} \sum_{j=0}^{k} \alpha_{j}^{(n)} \alpha_{k-j}^{(m)} a^{j}(1-a)^{k-j} .
$$

Recall the connection formula due to Carlitz [5]:

$$
u^{k}=\sum_{i=0}^{k} \delta_{i}^{(k)} q_{i}(u), \quad k=0,1, \ldots,
$$

where

$$
\delta_{i}^{(k)}=\left\{\begin{array}{cl}
\frac{(k+1) !}{2^{k}} \frac{(-1)^{k-i}(2 i) !}{(k-i) ! i !(2 i+1-k) !} & \text { for } \quad \frac{k-1}{2} \leq i \leq k, \\
0 & \text { for } 0 \leq i<\frac{k-1}{2} .
\end{array}\right.
$$

Plugging (13) into (12) and extracting the coefficient of $q_{i}(u)$ we obtain

$$
\beta_{i}^{(n, m)}(a)=\sum_{k=i}^{m+n} \delta_{i}^{(k)} \sum_{j=0}^{k} \alpha_{j}^{(n)} \alpha_{k-j}^{(m)} a^{j}(1-a)^{k-j} .
$$

Replacing $k$ by $k+i$ and $j$ by $n-j$ we obtain

$$
\begin{aligned}
\beta_{i}^{(n, m)}(a)= & \sum_{k=0}^{i+1} \delta_{i}^{(k+i)} \sum_{j=0}^{n} \alpha_{n-j}^{(n)} \alpha_{k+i-n+j}^{(m)} a^{n-j}(1-a)^{k+i-n+j} \\
= & \frac{(2 i) !}{i !}(1-a)^{i-n} \sum_{k=0}^{i+1} \sum_{j=0}^{n} \frac{(-1)^{k}(k+i+1) !}{k !(i+1-k) !} \\
& \times \frac{(-n)_{n-j}(-m)_{k+i-n+j} a^{n-j}(1-a)^{k+j}}{(-2 n)_{n-j}(n-j) !(-2 m)_{k+i-n+j}(k+i-n+j) !} .
\end{aligned}
$$

As $\beta_{i}^{(n, m)}(a)=0$ if $i<n$, replacing $i$ by $n+m-i$ and assuming $i \leq m$ we obtain, after some simplification,

$$
\begin{aligned}
\beta_{n+m-i}^{(n, m)}(a) & =4^{-i} \frac{(1 / 2)_{n+m-i}}{(1 / 2)_{n}(1 / 2)_{m}} a^{n+i}(1-a)^{m-i} \frac{(m+i) !}{(m-i) ! i !} \\
& \times \sum_{k \geq 0}(-1)^{k} \frac{(m+n-i+2)_{k}(-n-m+i-1)_{k}}{k !} \\
& \times \sum_{j \geq 0} \frac{(-n)_{j}(-i)_{k+j}(n+1)_{j} a^{-i+k}(1-1 / a)^{k+j}}{j !(-m-i)_{k+j}(1+m-i)_{k+j}} .
\end{aligned}
$$

By binomial formula, we have

$$
a^{-i+k}(1-1 / a)^{k+j}=\sum_{t \geq 0} \frac{(-k-j)_{t}}{t !} a^{-i+k-t} .
$$


Let $l=k+j$ and $r=i+t-k$ we can write

$$
\begin{aligned}
\beta_{n+m-i}^{(n, m)}(a) & =\frac{(1 / 2)_{n+m-i}(1 / 2)_{i}}{(1 / 2)_{n}(1 / 2)_{m}} a^{n+i}(1-a)^{m-i}\left(\begin{array}{c}
m+i \\
2 i
\end{array}\right) \\
& \times \sum_{r=0}^{2 i} a^{-r} \sum_{l \geq 0} \frac{(-i)_{l}(-n)_{l}(n+1)_{l}}{(-m-i)_{l}(1+m-i)_{l} l !} \\
& \times \sum_{k \geq 0} \frac{(-l)_{k}(-l)_{r-i+k}(m+n-i+2)_{k}(-n-m+i-1)_{k}}{k !(r-i+k) !(n-l+1)_{k}(-n-l)_{k}} .
\end{aligned}
$$

For $i=0,1$ and 2, the above formula yields immediately the following explicit expressions:

$$
\begin{gathered}
\beta_{n+m}^{(n, m)}(a)=a^{n}(1-a)^{m} \frac{(1 / 2)_{n+m}}{(1 / 2)_{n}(1 / 2)_{m}}, \\
\beta_{n+m-1}^{(n, m)}(a)=a^{n+1}(1-a)^{m-1} \frac{(1 / 2)(1 / 2)_{n+m-1}}{(1 / 2)_{n}(1 / 2)_{m}} \\
\times\left(\left(\begin{array}{c}
n+m+1 \\
2
\end{array}\right)-\left(\begin{array}{c}
n+m+1 \\
1
\end{array}\right)\left(\begin{array}{c}
n \\
1
\end{array}\right) a^{-1}+\left(\begin{array}{c}
n+1 \\
2
\end{array}\right) a^{-2}\right),
\end{gathered}
$$

and

$$
\begin{aligned}
\beta_{n+m-2}^{(n, m)}(a) & =a^{n+2}(1-a)^{m-2} \frac{(1 / 2)_{2}(1 / 2)_{n+m-2}}{(1 / 2)_{n}(1 / 2)_{m}} \\
& \times\left(\left(\begin{array}{c}
m+n+1 \\
4
\end{array}\right)-\left(\begin{array}{c}
n+m+1 \\
3
\end{array}\right)\left(\begin{array}{c}
n-1 \\
1
\end{array}\right) a^{-1}\right. \\
& +\left(\begin{array}{c}
n+m+1 \\
2
\end{array}\right)\left(\begin{array}{c}
n \\
2
\end{array}\right) a^{-2}-\left(\begin{array}{c}
n+m+1 \\
1
\end{array}\right)\left(\begin{array}{c}
n+1 \\
3
\end{array}\right) a^{-3} \\
& \left.+\left(\begin{array}{c}
n+2 \\
4
\end{array}\right) a^{-4}\right) .
\end{aligned}
$$

Further computation led us to conjecture the formula (66), which is equivalent to (7) and (8).

Our proof consists of verifying that the conjectured formula (6) does satisfy the boundary condition (4) and the recurrence relation (5).

2.2. The boundary condition. We first show that the formula (6) reduces to (4) when $m=0$. Let $c_{k}^{(n)}(a)=\beta_{k}^{(n, 0)}(a)$ for $0 \leq k \leq n$. Clearly we have $c_{n}^{(n)}(a)=a^{n}$ from (6). Assume that $0 \leq k \leq n-1$, using the symmetry $c_{n}^{(n)}(a)=\beta_{k}^{(0, n)}(1-a)$, we derive from (6) that

$$
\begin{aligned}
c_{k}^{(n)}(a) & =(1-a) a^{k}(1 / 2)_{n-k} \frac{(1 / 2)_{k}}{(1 / 2)_{n}} \\
& \times \sum_{j=0}^{2 n-2 k}(-1)^{j}\left(\begin{array}{c}
n+1 \\
2 n-2 k-j
\end{array}\right)\left(\begin{array}{c}
k-n+j \\
j
\end{array}\right)(1-a)^{n-k-j-1} .
\end{aligned}
$$


As $\left(\begin{array}{c}k-n+j \\ j\end{array}\right)=0$ if $j \geq n-k$, replacing $j$ by $n-k-1-j$ we obtain

$$
\begin{aligned}
c_{k}^{(n)}(a)= & (1-a) a^{k}(1 / 2)_{n-k} \frac{(1 / 2)_{k}}{(1 / 2)_{n}} \\
& \times \sum_{j=0}^{(n-k-1) \wedge k}(-1)^{n-k-1-j}\left(\begin{array}{c}
n+1 \\
n-k+1+j
\end{array}\right)\left(\begin{array}{c}
-1-j \\
n-k-1-j
\end{array}\right)(1-a)^{j} .
\end{aligned}
$$

Since

$$
(1 / 2)_{n-k} \frac{(1 / 2)_{k}}{(1 / 2)_{n}}=\frac{\left(\begin{array}{l}
n \\
k
\end{array}\right)}{\left(\begin{array}{l}
2 n \\
2 k
\end{array}\right)}, \quad\left(\begin{array}{c}
n+1 \\
n-k+1+j
\end{array}\right)=\left(\begin{array}{c}
n+1 \\
k-j
\end{array}\right),
$$

and

$$
\begin{aligned}
(-1)^{n-k-1-j}\left(\begin{array}{c}
-1-j \\
n-k-1-j
\end{array}\right) & =\frac{(j+1)(j+2) \ldots(n-k-1)}{(n-k-1-j) !} \\
& =\left(\begin{array}{c}
n-k-1 \\
j
\end{array}\right),
\end{aligned}
$$

we derive the desired formula (41).

2.3. The recurrence relation. It remains to verify (5) for the conjectured formula (6). One of Gauss' relation for contiguous hypergeometric functions [7, p. 71] reads:

$$
\begin{aligned}
& C_{2} F_{1}\left(\begin{array}{c}
A, B \\
C
\end{array} ;\right) \\
& =(C-A) z_{2} F_{1}\left(\begin{array}{c}
A, B+1 \\
C+1
\end{array} ; z\right)+C(1-z){ }_{2} F_{1}\left(\begin{array}{c}
A, B+1 \\
C
\end{array} ; z\right) .
\end{aligned}
$$

This can also be verified straightforwardly by comparing the coefficients of $z^{k}$ for each $k \in \mathbb{N}$. Similarly we have the following contiguous relation:

$$
\begin{aligned}
& B(1-z){ }_{2} F_{1}\left(\begin{array}{c}
A, B+1 \\
C+1
\end{array} ; z\right) \\
& =(B-C) z_{2} F_{1}\left(\begin{array}{c}
A, B \\
C+1
\end{array} ; z\right)+C_{2} F_{1}\left(\begin{array}{r}
A-1, B \\
C
\end{array} ; z\right) .
\end{aligned}
$$

Now, the recurrence relation (5) is satisfied by the conjectured formula (6) since

- for $k \geq\lceil(n+m-1) / 2\rceil$, this is equivalent to (18) with $z=1 / a$ and $A=-2 m-2 n+2 k+2, \quad B=-m+k+1, \quad C=-n-$ $m+3+2 k$.

- for $k \leq\lfloor(n+m-1) / 2\rfloor$, this is equivalent to (19) with $z=1 / a$ and $A=-m-n, \quad B=-n-k-1, \quad C=n-m-2 k$. 
The formula is thus proved.

As a byproduct, comparing (66) and (17) we derive immediately the following double sum identity.

Corollary 2. For all positive integers $i, m, n$ and $r=0,1, \ldots, 2 i$ we have

$$
\begin{aligned}
\left(\begin{array}{c}
m+i \\
2 i
\end{array}\right) & \sum_{l=\max \{0, i-r\}}^{\min \{i, n\}} \sum_{k=\max \{0, i-r\}}^{\min \{l, l+i-r\}} \frac{(-i)_{l}(-n)_{l}(n+1)_{l}}{(-m-i)_{l}(1+m-i)_{l} l !} \\
& \times \frac{(-l)_{k}(-l)_{r-i+k}(m+n-i+2)_{k}(-n-m+i-1)_{k}}{k !(r-i+k) !(n-l+1)_{k}(-n-l)_{k}} \\
& =(-1)^{r}\left(\begin{array}{c}
m+n+1 \\
2 i-r
\end{array}\right)\left(\begin{array}{c}
n-i+r \\
r
\end{array}\right) .
\end{aligned}
$$

It would be interesting to find a direct standard proof of (201) using the hypergeometric function theory.

\section{Applications}

3.1. A positivity result. Theorem 1 implies immediately the following positivity result.

Corollary 3. Suppose that $n<m$. Then the coefficient $\beta_{k}^{(n, m)}(a)$ is positive for $n \leq k<m$.

Proof. For $(n+m-1) / 2 \leq k<m$, we have $-m+k+1 \leq 0,-2 m-$ $2 n+2 k<0$ and $2 k-m-n+2>0$. Hence each summand in (77) is positive and so is $\beta_{k}^{(n, m)}(a)$. For $n \leq k \leq(n+m-1) / 2$, we derive from (8) and (10) that

$$
\begin{aligned}
\beta_{k}^{(n, m)}(a) & =a^{k-m}(1-a)^{m+k+1} \frac{(1 / 2)_{n+m-k}(1 / 2)_{k}}{(1 / 2)_{n}(1 / 2)_{m}} \\
& \times\left(\begin{array}{c}
n-k-1 \\
n+m-2 k-1
\end{array}\right){ }_{2} F_{1}\left(\begin{array}{c}
-m-n-1, \quad m-k \\
n+m-2 k
\end{array} ; \frac{1}{1-a}\right) .
\end{aligned}
$$

In this case, we have $-m-n-1<0, m-k>0$ and $m+n-$

$2 k>0$, therefore each summand in (21) is positive and so is $\beta_{k}^{(n, m)}(a)$. Summarizing the above two cases, we obtain the desired positivity.

3.2. A formula of Berg and Vignat. Let $\beta_{i}^{(n)}(a)$ be the coefficients defined by

$$
q_{n}(a u) q_{n}((1-a) u)=\sum_{i=0}^{n} \beta_{i}^{(n)}(a) q_{n+i}(u)
$$


Corollary 4. For $0 \leq i \leq n$ we have

$$
\begin{aligned}
\beta_{i}^{(n)}(a) & =\frac{(4 a(1-a))^{i}}{4^{n}} \frac{(-n)_{i}\left(n+\frac{1}{2}\right)_{i}}{i !\left(-n+\frac{1}{2}\right)_{i}} \\
& \times{ }_{2} F_{1}\left(\begin{array}{c}
-n+i, \\
\frac{1}{2}-n-\frac{1}{2}
\end{array} ;(2 a-1)^{2}\right) .
\end{aligned}
$$

Proof. By definition we have $\beta_{i}^{(n)}(a)=\beta_{n+i}^{(n, n)}(a)$. It follows from (6) that

$$
\begin{aligned}
\beta_{i}^{(n)}(a)=a^{2 n-i}(1-a)^{i}(1 / 2)_{n-i} \frac{(n+1 / 2)_{i}}{(1 / 2)_{n}}\left(\begin{array}{c}
2 n+1 \\
2 n-2 i
\end{array}\right) \\
\times{ }_{2} F_{1}\left(\begin{array}{c}
-2 n+2 i, \quad i+1 \\
2 i+2
\end{array} ; \frac{1}{a}\right) .
\end{aligned}
$$

Applying the quadratic transformation formula [1, p. 127] :

$$
{ }_{2} F_{1}\left(\begin{array}{c}
a, b \\
2 a
\end{array} ; x\right)=(1-x / 2)_{2}^{-b} F_{1}\left(\begin{array}{c}
b / 2, \underset{ }{(b+1) / 2} \\
a+1 / 2
\end{array} ;\left(\frac{x}{2-x}\right)^{2}\right),
$$

we obtain

$$
\begin{aligned}
\beta_{i}^{(n)}(a)=a^{2 n-i}(1-a)^{i}(1 / 2)_{n-i} \frac{(n+1 / 2)_{i}}{(1 / 2)_{n}}\left(\begin{array}{c}
2 n+1 \\
2 n-2 i
\end{array}\right) \\
\quad \times\left(\frac{2 a-1}{2 a}\right)^{2 n-2 i}{ }_{2} F_{1}\left(\begin{array}{c}
i-n, \begin{array}{c}
i-n-1 / 2 \\
i+3 / 2
\end{array} ;(2 a-1)^{-2}
\end{array}\right) .
\end{aligned}
$$

Reversing the order of summation in the last ${ }_{2} F_{1}$ and using $(x)_{n-k}=$ $(-1)^{k} \frac{(x)_{n}}{(-x-n+1)_{k}}$ we recover (23) .

3.3. An integral evaluation. Recall [2] the Student $t$-distribution with parameter $\nu>0$ :

$$
f_{\nu}(x)=\frac{A_{\nu}}{\left(1+x^{2}\right)^{\nu+\frac{1}{2}}}, \quad A_{\nu}=\frac{\Gamma\left(\nu+\frac{1}{2}\right)}{\Gamma\left(\frac{1}{2}\right) \Gamma(\nu)} .
$$

For $\nu=n+\frac{1}{2}$ with $n \in \mathbb{N}$ we say that the distribution has $2 n+1$ degree of freedom. The Fourier transform of the density $f_{\nu}$ is an even function, which for $\xi \geq 0$, can be expressed as

$$
k_{\nu}(\xi)=\int_{-\infty}^{\infty} f_{\nu}(x) e^{-i x \xi} d x=\frac{2^{1-\nu}}{\Gamma(\nu)} \xi^{\nu} K_{v}(\xi)
$$

where $K_{\nu}$ is the modified Bessel function of the third kind, also called the Macdonald function.

For $\nu=n+\frac{1}{2}$ it is known that $k_{\nu}(u)=e^{-u} q_{n}(u)$ for $u \geq 0$. Thus equation (3) is equivalent to

$$
\frac{1}{a} f_{n+\frac{1}{2}}\left(\frac{x}{a}\right) * \frac{1}{1-a} f_{m+\frac{1}{2}}\left(\frac{x}{1-a}\right)=\sum_{k=n \wedge m}^{n+m} \beta_{k}^{(n, m)}(a) f_{k+\frac{1}{2}}(x),
$$


where $0<a<1$ and $*$ is the ordinary convolution of densities.

For $a=\frac{1}{2}$, replacing $x$ by $\frac{x}{2}$ and multiplying by $\frac{1}{2}$ on both sides, we obtain

$$
f_{n+\frac{1}{2}} * f_{m+\frac{1}{2}}(x)=\sum_{k=n \wedge m}^{n+m} \frac{1}{2} \beta_{k}^{(n, m)}\left(\frac{1}{2}\right) f_{k+\frac{1}{2}}\left(\frac{x}{2}\right),
$$

which is equivalent to the following integral evaluation.

$$
\int_{-\infty}^{+\infty} \frac{d y}{\left(1+y^{2}\right)^{n+1}\left(1+(x-y)^{2}\right)^{m+1}}=\frac{1}{2} \sum_{k=m \wedge n}^{m+n} \frac{A_{k+\frac{1}{2}}}{A_{n+\frac{1}{2}} A_{m+\frac{1}{2}}} \frac{\beta_{k}^{(n, m)}\left(\frac{1}{2}\right)}{\left(1+\frac{1}{4} x^{2}\right)^{k+1}} .
$$

By Theorem 1 with $a=1 / 2$, the above formula can be simplified as follows.

Corollary 5. For positive integers $m$ and $n$ we have

$$
\int_{-\infty}^{+\infty} \frac{n ! m ! 2^{n+m} d y}{\left(1+y^{2}\right)^{n+1}\left(1+(x-y)^{2}\right)^{m+1}}=\frac{\pi}{2} \sum_{k=m \wedge n}^{m+n} \frac{\gamma_{k}^{(n, m)}}{\left(1+\frac{1}{4} x^{2}\right)^{k+1}},
$$

where

$$
\gamma_{k}^{(n, m)}=\sum_{j=0}^{2(n+m-i)}(-2)^{j}\left(\begin{array}{c}
n+m+1 \\
2 n+2 m-2 i-j
\end{array}\right)\left(\begin{array}{c}
-m+i+j \\
j
\end{array}\right) .
$$

When $m=n$, as shown by Berg and Vignat [3], the above formula yields a new proof of an integral formula of Boros and Moll [4].

Acknowledgement. This work was done mainly during the first author's visit to Université Lyon 1 in spring 2011 and supported by a scholarship "Séjours Scientifiques de Haut Niveau (SSHN)" de l'Institut français de Tunisie.

\section{REFERENCES}

[1] G. Andrews, R. Askey, R. Roy, Special functions, Encyclopedia of Mathematics and its applications 71, Cambridge University Press, Cambridge, 1999.

[2] C. Berg and C. Vignat, Linearization coefficients of Bessel polynomials and properties of Student $t$-Distributions, Constructive Approximation, 27 (2008), $15-32$.

[3] C. Berg and C. Vignat, Derivation of an integral of Boros and Moll via convolution of Student $t$-densities, to appear in Ramanujan J. DOI 10.1007/s11139010-9279-5.

[4] G. Boros and V. H. Moll, An integral hidden in Gradshteyn and Ryzhik, J. Comput. Appl. Math. 106 (1999), 361-368.

[5] L. Carlitz, A note on the Bessel polynomials, Duke Math. J., 24 (1957), 151162.

[6] E. Grosswald, Bessel polynomials, Lecture Notes in Mathematics 698, Springer, New York 1978.

[7] E. D. Rainville, Special Funtions, Macmillan Company, New York, 1960. 
(Mohamed Jalel Atia) Faculté de Science, Université de Gabès, Tunisie E-mail address: jalel.atia@gmail.com

(Jiang Zeng) Université de Lyon; Université Lyon 1; Institut Camille Jordan; UMR 5208 DU CNRS; 43, BOULEVARD DU 11 NOVEMBRE 1918, F69622 Villeurbanne Cedex, France

E-mail address: zeng@math.univ-lyon1.fr 